\title{
Building capacity in systematic review searching: a pilot program using virtual mentoring ${ }^{1,2}$
}

\author{
Trina Fyfe and Liz Dennett
}

\begin{abstract}
Introduction: A well thought out, comprehensive search is the foundation for all systematic review research. Consequently, working on a first systematic review search can be intimidating. Articles and workshops, being less intensive than one-on-one training, are unlikely to instil enough confidence in one's ability to do a good job in this process. In this pilot program, an ongoing mentoring relationship was created as a means of building capacity and confidence in systematic review searching. Description: The mentorship program occurred in a virtual environment because the mentee and mentor were at two different institutions. Outcomes: To compensate for the distance, the mentor and mentee communicated and collaborated using free online collaborative software. The process for engaging in the program was developed between both the mentor and mentee to ensure individual and shared objectives would be met. This pilot program's process involved sharing searches, reflective journaling and recording time. Discussion: Building capacity in systematic review searching with an inexperienced searcher through virtual mentoring is an effective means of building confidence and skills in systematic review searching, and in building a sustainable mentor/mentee relationship.
\end{abstract}

\section{Introduction}

Getting involved with a systematic review project can be intimidating for a librarian who has never had the opportunity to work on one before. The librarian is usually responsible for creating the search strategy, which ideally should be comprehensive, error-free, unbiased (i.e. include published and unpublished research) and accurately documented. McGowan and Sampson state emphatically: "Librarians who conduct the searches for systematic reviews must be experts [1]." This expertise is required because the search is the foundation of a systematic review; errors made in the search can result in important evidence being missed, thus affecting the quality of the review and its conclusions.

So how should a health sciences librarian with no experience in systematic review searching ensure that he/ she has the necessary knowledge and skills before offering information services to a systematic review team? While there are some systematic review search training sessions available and a number of books and articles that provide guidance (notably the Cochrane Handbook for Systematic Reviews of Interventions [2]), it can still be difficult to feel confident that one has acquired the requisite competencies. While expert searching and the competencies involved has been well-defined by the MLA policy statement [3] on the topic, there is limited research on how librarians actually acquire expert searching knowledge and what training methods can be used to improve their searches. Indeed, Holst and Funk mention the dearth of research surrounding the entire concept of "expertness" in librarianmediated searching [4]. The vast majority of literature on the topic consists of opinion pieces. In one small study, Vieira and Dunn conducted a survey of librarians who viewed each other's search strategies through a common email. All of the librarians agreed they sought help from their colleagues to improve searches and improved their own searches by reading each other's answers [5]. Additionally, the MLA Task Force on Expert Searching surveyed MLA members on topics related to "expert searching." Of the 256 respondents, $83.1 \%$ said they felt their search skills had improved in the past 10 years. The top two reasons given for improvement were experience $(62.7 \%)$ and training (59.8\%). Networking with colleagues, improvement in technology, and reading the literature were also found to be helpful [4]. Following a program that

T. Fyfe ${ }^{3}$. MISt, Northern Medical Program, University of Northern British Columbia, 3333 University Way, Prince George BC, V2N 4Z9, Canada.

L. Dennett. MLIS, John W Scott Health Sciences Library, University of Alberta and the Institute of Health Economics, Edmonton AB T6G 2R7, Canada.

${ }^{1}$ Based on presentation at: Canadian Health Libraries Association Conference, Winnipeg, MB, 2 June 2009.

${ }^{2}$ This article has been peer reviewed.

${ }^{3}$ Corresponding author (e-mail: fyfet@unbc.ca). 
contains both experience and training, in addition to contact with an experienced searcher, may be a good way to acquire expert searching skills.

Mentoring may also be useful for developing skills. In a study conducted by the South Central Chapter of the Medical Library Association on mentoring [4], 71\% of the respondents with mentors considered it a critical part of their professional experience. The respondents also indicated the most important mentoring activity was skills development that improved job performance [6]. In many organizations, formal or informal mentoring or training to develop librarians' skills in systematic searching likely already occurs. However, if the librarian desiring to improve her skills is a solo librarian and (or) geographically isolated from other experienced systematic review searchers, traditional mentoring proves challenging.

This pilot program began when a solo, geographically isolated health librarian from a small research university was asked by a researcher if she could assist with a systematic review project. The librarian had not worked on one before and although she had attended a half-day training session on systematic review searching, she still felt unprepared. She mentioned these concerns (during a conversation at a CHLA conference) to a health librarian from a large institution who regularly worked on systematic reviews. Together they decided that this was likely a common problem and that they would try to come up with a model for a librarian to get experience in the systematic review process and then pilot the model, using the solo librarian as the first beneficiary. Both parties obtained approval from their institutions to participate in this project.

\section{Description}

During an initial phone meeting, goals and desired learning outcomes of the project, as well as logistics, were discussed. The librarian from the small research institution, (henceforth referred to as the mentee) was already a knowledgeable health information searcher. Her goal was to build capacity in systematic review searching and thus gain the confidence needed to work with systematic review teams at her own institution. The experienced systematic review searcher (henceforth referred to as the mentor) suggested employing a method that had been successfully used multiple times at the University of Alberta John W. Scott Health Sciences Library to teach new health librarians the skills needed to participate in systematic review projects. The method involves an experienced systematic review searcher and a novice librarian working together as part of a systematic review team. They attend all meetings with the systematic review team, co-create and jointly carry out the search strategy and work together on providing the results to the researchers. The experienced searcher provides mentoring and training throughout the experience.

For this pilot program, the mentor and mentee initially tried to arrange for the mentee to visit the mentor's institution in order to replicate the mentorship program used at the University of Alberta's John W. Scott Health Sciences Library. Funding from the mentee's department was obtained. However, the participants quickly realized that it would be very difficult for the mentee to arrange her schedule in such a way that she could drop all her other responsibilities when a suitable systematic review project was initiated at the mentor's institution. The participants realized that this challenge was actually an opportunity to pilot the feasibility of virtual mentoring for skill development, and that using virtual mentoring was a model that was more likely to be adapted by others with similar professional development needs. Eldredge champions the benefits of virtual peer mentoring to help people learn to incorporate the evidence-based librarianship process in their decision making [7] and the mentor and mentee felt that it could also be used in a similar way to gain confidence in systematic searching.

Virtual mentoring usually employs phone or email as its main technology, but in this case a more sophisticated technology was sought in order to more closely mimic the process of working together in person. The participants decided that the free version of the web conferencing program Elluminate (recently changed to Blackboard Collaborate) was ideal as it offered the opportunity to talk to each other and share desktops. Zoho Docs, which offers online document editing, was used to store the project documents so that the latest versions would be available to both mentor and mentee and allowed for coasynchronous editing. Early on it was decided that both participants would keep track of the time they dedicated to the project so that interested individuals seeking to replicate the program would have an idea of the amount of time they could expect to spend.

While ideally the mentee would have participated in the entire process of conducting a systematic review with an active systematic review project and team, the opportunity did not present itself during the project period. It was decided that working on an in-process systematic review would be similarly effective and such a project was identified by the mentor. The mentor provided the mentee with the original research question and they role played the initial reference interview (with the mentor acting in the role of researcher) in order to construct a focused and searchable question. Then the mentee conducted and recorded her own MEDLINE search without seeing the mentor's already completed search and recorded any questions or difficulties that she experienced in a reflective journal. Reflective journaling has been shown to provide "a medium through which learners can identify viable topics for concern, and reflect critically on their experiences" and "a means of establishing and maintaining a relationship between coach and client" [8]. To this end, the mentee recorded her thoughts and questions as she worked through the search and posted her journal on Zoho for the mentor to read. The mentor also kept a journal of her reflections each time they met to discuss the systematic review project.

Once the mentee had completed her MEDLINE search, the mentor reviewed the mentee's journal entry, and compared the mentee's search to her own search and identified concerns with the search terms or methodology. They then met virtually using Elluminate and reviewed the search line by line, worked through the mentee's questions, 
and talked about the problems the mentor identified. They also discussed any variations between the mentor and mentee's search strategies and merged the two searches to create an "ideal" version that included the best of both strategies. Finally the mentor shared the list of databases and websites that she usually searches for a systematic review. To prepare for the next meeting, the mentee was asked to translate the MEDLINE search strategy to the other databases identified (i.e. Cochrane Library, Pubmed, CRD Databases, EMBASE, CINAHL, PsycINFO, Academic Search Premier, Sociological Abstracts, SOCIndex, Social Services Abstracts, Web of Science, CBCA Reference) and also search the grey literature.

During the next meeting, the mentor and mentee reviewed the mentee's questions about searching the other databases and grey literature websites, and a joint search strategy was finalized.

\section{Outcomes}

In total, the participants met via Elluminate for 4.75 hours to discuss the searches. The mentor spent an additional 2.5 hours reviewing the mentee's search strategy before the meetings. The additional amount of time the mentor spent mentoring and training on this project was approximately 7.25 hours.

Admittedly our main outcome of concern (i.e. having the confidence to participate as a solo librarian in future systematic review teams) is a fairly subjective outcome. As stated earlier, the main outcome of interest for the project was whether or not the mentee would feel confident to develop a search on her own on any new systematic review project. The mentee's reflective journal noted that in addition to learning a number of useful searching tips from the mentor, she had gained confidence in her existing search skills because her mentor was able to tell her that much of her search was very well executed. She now feels ready to be part of a new systematic review team, in part because she knows that if any difficulties or questions arise, she can always ask her mentor. The mentor also found the project a valuable use of her time. She recorded in her reflective journal her enjoyment of sharing her expertise and she reflected on how the project made her aware of how much she had learned since starting to work on systematic reviews. The mentor also noted that the mentee had come up with some useful search terms that the mentor had missed in her original search.

Indeed, together, the mentor and mentee's combined search ended up being more comprehensive because of the mentee's additions. And this improved version of the search was used to update the actual systematic review project before publication so that the quality of the systematic review also benefitted from this pilot program.

A final positive outcome was that the participants developed a "tip sheet" (Appendix A) to provide a stepby-step outline of the project and some suggestions for best practice to guide anyone interested in replicating this type of capacity-building exercise.

\section{Discussion}

Applying a virtual mentoring model in this pilot program was a success. Technology was pivotal in overcoming the geographic barrier between the mentor and mentee and for keeping project costs to a minimum. However, exploring, evaluating, and learning to use programs takes time. If people are considering replicating this pilot and have never used web conferencing software, they should expect to spend some additional time familiarizing themselves with the technology before the project starts.

Agreeing to be a mentor may be daunting. It is hoped that our time estimate of less than eight hours of additional work will encourage potential mentors that the time commitment does not have to be burdensome, particularly if the search they are working on is part of the mentor's normal job responsibilities.

The reflective journaling the participants used highlighted some successes and learning opportunities for future virtual mentorship programs. The mentee's reflective journal demonstrated the mentee's growth and confidence in systematic review searching. As Gray states, "Writing a reflective journal sensitizes self to self and enables those providing feedback and help, such as a coach, to become sensitive "within the moment" [8]. The narrative experiences of the mentee were helpful for the mentor to see progress, frustration, need for clarification, and other learning opportunities not identified at the outset of the project. The entries provided a good base for discussion in virtual meetings. The reflective journal entries were a great tool for the mentee to reflect upon at the end of the project as she could see the progress that was made along the way and was assured that learning outcomes were achieved.

This is only a single instance of virtual mentoring to build capacity in systematic review searching. It is hoped that the mentee's success will inspire others, who lack confidence or the searching skills required to take on a systematic review project, to find qualified mentors to guide them through the process. (Mentors can be found through programs such as the CHLA/ABSC Mentorship Program, workplace programs, or by asking an experienced librarian if they would be interested.) The model presented herein could be adapted for different environments, both physical and virtual, and for different areas of skill development.

\section{Conclusion}

Mentorship proved to be a successful means of helping a novice systematic review searcher to build confidence and increase expert searching knowledge. Although our initial goal of working together from the very start of a systematic review project was not realized, the mentor tried to replicate the experience of working with the researchers at the initial project meeting, and the lack of pressing timelines allowed time for much discussion and reflection. Since the systematic review project was ongoing, the mentor and mentee's combined search was able to be 
used for the project's update search and in the final published report. This virtual mentoring pilot program of fostered respectful, meaningful, effective, and sustainable interactions between the mentor and mentee. The appended tip sheet should help other health librarians successfully replicate this program.

\section{References}

1. McGowan J, Sampson M. Systematic reviews need systematic searchers. J Med Libr Assoc. 2005;93(1):74-80.

2. Higgins, JPT, Green S (editors). Cochrane Handbook for Systematic Reviews of Interventions. Version 5.1.0 [updated March 2011]. The Cochrane Collaboration, 2011. Available from www cochrane-handbook org

3. Medical Library Association. Role of expert searching in health sciences libraries. $J$ Med Libr Assoc. 2005;93(1):42-4.
4. Holst R, Funk CJ. State of the art of expert searching: results of a Medical Library Association survey. J Med Libr Assoc. 2005;93(1):45-52.

5. Vieira DL, Dunn K. Peer training in expert searching: the observation effect. J Med Libr Assoc. 2005;93(1):69-73.

6. Kwasik H, Fulda PO. Strengthening professionals: a chapterlevel formative evaluation of the Medical Library Association mentoring initiative. J Med Libr Assoc. 2006;94(1):19-29.

7. Eldredge JD. Virtual Peer Mentoring (VPM) may facilitate the entire EBLIP process. Evid Based Libr \& Inf Pract. 2010;5(1):7-16.

8. Gray DE. Facilitating Management Learning: developing critical reflection through reflective tools. Manag Learn. 2007;38(5):495-517. doi:10.1177/1350507607083204. 


\section{Appendix A: Building Capacity in Systematic Review Searching Tip Sheet}

The following steps assume that the "mentee" is experienced with searching the medical literature but has little or no experience in systematic review searching.

1) Mentor and mentee should meet to discuss the mentee's learning objectives.

2) A new or ongoing project needs to be identified by either the mentor or mentee. Ideally the project should not have very tight timelines as it may be difficult to make time for both searching and meeting to talk about and compare search results. Alternatively, a completed search can be used to provide an example topic for the mentor and mentee to work through, particularly if no appropriate new project is identified.

3) If the project is active, both librarians should meet (virtually or in person) with the researcher(s) to determine scope and terms of the systematic review project.

4) If you are working together virtually, become familiar with some technologies that will enable you to share documents and desktops.

5) Mentor and mentee should each complete a MEDLINE search. Mentee should keep track of any questions and (or) problems while searching MEDLINE.

6) Mentee can submit search and questions and (or) problems for mentor to look over before they meet. Then they can meet to go over the search together.

7) Combine searches into one agreed upon "ideal" MEDLINE search strategy. They should also discuss which additional databases / websites / grey literature sources / journals will be searched. It is also good to discuss how search strategy will be documented.

8) The mentee and mentor can then each adapt the search for the rest of the databases, websites, etc. It is important to remember that sometimes new search terms can come to light while searching other databases that may be used to improve the original MEDLINE search. Both searchers should be on the lookout for these and include them in the search where appropriate. Again, the mentee should note any questions they have while doing these searches.

9) Mentor and mentee should meet again to share and discuss these searches and go through the mentee's questions, ensuring that the mentee's learning objectives have been met and the mentee feels confident of his or her systematic review searching competencies.

10) One or both of the librarians (depending on time) can merge the searches for optimal retrieval and can download the results into citation databases for the researchers (if working on an active project).

Remember to maintain the mentoring relationship so that the mentee feels comfortable asking questions during his or her first solo systematic review search. 